Proyecciones Journal of Mathematics

Vol. 37, No 3, pp. 583-591, September 2018.

Universidad Católica del Norte

Antofagasta - Chile

\title{
A new type of difference class of interval numbers
}

\author{
Achyutananda Baruah \\ North Gauhati College, India \\ and \\ Amar Jyoti Dutta \\ Pragjyotish College, India \\ Received : January 2018. Accepted : January 2018
}

\begin{abstract}
In this article we introduce the notation difference operator $\Delta_{m}$ ( $m \geq 0$ be an integer) for studying some properties defined with interval numbers. We introduced the classes of sequence $\bar{\ell}(p)\left(\Delta_{m}\right), \bar{c}(p)\left(\Delta_{m}\right)$ and $\bar{c}_{0}(p)\left(\Delta_{m}\right)$ and investigate different algebraic properties like completeness, solidness, convergence free etc.
\end{abstract}

Key Words : Interval number, Completeness, Solid, Convergence free.

AMS Subject Classification : 40C05, 40J05, 46A45. 


\section{Introduction}

The concept of interval arithmetic was first suggested by Dwyer [15] in 1951. Thereafter the concept has been using in area of science and technology. The evidence of its development as a formal system and application in computational device is found in Moore [8], Moore and Yang [9] and others ([15], [16], [17] and [20]). Different mathematical concepts were introduced and studied with interval numbers by several researchers across the globe. Chiao [13] introduced sequence of interval numbers and defined usual convergence of sequences of interval number. Sengnl and Eryilmaz [14] introduced and studied bounded and convergent sequence spaces of interval numbers and proved that these spaces are complete metric space. Recently Esi [1-8], Esi and Braha [18], Esi and Esi [19], Esi and Hazarika [20] and Esi and Catalbas [21] introduced and studied strongly almostconvergence and statistically almost-convergence of interval numbers.

A set consisting of a closed interval of real numbers $x$ such that $a \leq$ $x \leq b$ is called an interval number. A real interval can also be considered as a set. We can investigate some properties of interval numbers, for instance arithmetic properties or analysis properties. We denote the set of all real valued closed intervals by $R$. Any elements of $R$ is called closed interval and denoted by $\bar{x}$, that is $\bar{x}=\{x \in R: a \leq x \leq b\}$. An interval number $\bar{x}$ is a closed subset of real numbers [15]. Let $x_{l}$ and $x_{r}$ be first and last points of interval number $\bar{x}$, respectively then we have for $x_{1}, x_{2} \in R$,

i) $\overline{x_{1}}=\overline{x_{2}} \Leftrightarrow x_{1_{\ell}}=x_{2_{\ell}}, x_{1_{r}}=x_{2_{r}}$

ii) $\overline{x_{1}}+\overline{x_{2}}=\left\{x \in R: x_{1_{\ell}}+x_{2_{\ell}} \leq x \leq x_{1_{r}}+x_{2_{r}}\right\}$

iii) $\alpha \bar{x}=\left\{x \in R: \alpha x_{1_{\ell}} \leq x \leq \alpha x_{1_{r}}\right\}$, for $\alpha \geq 0$.and $\alpha x=\{x \in R$ : $\left.\alpha x_{1_{r}} \leq x \leq \alpha x_{1_{l}}\right\}$, for $\alpha<0$.

iv) $\overline{x_{1}} \overline{x_{2}}=\left\{x \in R: \min \left\{x_{1_{\ell}} x_{2_{\ell}}, x_{1_{\ell}} x_{2_{r}}, x_{1_{r}} x_{2_{l}}, x_{1_{r}} x_{2_{r}}\right\} \leq x\right.$

$$
\leq \max \left\{x_{1_{\ell}} x_{2_{\ell}}, x_{1_{\ell}} x_{2_{r}}, x_{1_{r}} x_{2_{\ell}}, x_{1_{r}} x_{2_{r}}\right\}
$$

The set of all interval numbers $R$ is a complete metric space defined by

$$
d\left(\overline{x_{1}}, \overline{x_{2}}\right)=\max \left\{\left|x_{1_{\ell}}-x_{2_{\ell}}\right|,\left|x_{1_{r}}-x_{2_{r}}\right|\right\}
$$



$R$.

In the special case, $\bar{x}_{1}=[a, a]$ and $\bar{x}_{2}=[b, b]$, we obtain usual metric of

Consider the transformation $f: N \rightarrow R$, by $k \rightarrow f(k)=\bar{x}, x=\left(x_{k}\right)$, then $\bar{x}=\left(\bar{x}_{k}\right)$ is called sequence of interval numbers. The term $\bar{x}_{k}$ is called the $k$ th term of sequence $(\bar{x})=\left(\bar{x}_{k}\right)$.

By $w^{i}$ we denotes the set of all interval numbers with real terms. We give the following definitions of convergence of interval numbers.

A sequence $\bar{x}=\left(\bar{x}_{k}\right)$ of interval numbers is said to be convergent to the interval number $\bar{x}_{0}$ if for each $\varepsilon>0$ there exists a positive integer $k_{0}$ such that $d\left(\bar{x}_{k}, \bar{x}_{0}\right)<\varepsilon$ for all $k \geq k_{0}$, denoted by $\lim _{k} \bar{x}_{k}=\bar{x}_{0}$. This imply that

$$
\lim _{k} \bar{x}_{k}=\bar{x}_{0} \Leftrightarrow \lim _{k} x_{k_{\ell}}=x_{0_{\ell}} \text { and } \lim _{k} x_{k_{r}}=x_{0_{r}} .
$$

An interval valued sequence space $\bar{E}$ is said to be solid if $\bar{y}=\left(\bar{y}_{k}\right) \in \bar{E}$ whenever $\left|\bar{y}_{k}\right| \leq\left|\bar{x}_{k}\right|$, for all $k \in N$ and $\bar{x}=\left(\bar{x}_{k}\right) \in \bar{E}$.

An interval valued sequence space $\bar{E}$ is said to be monotone if $\bar{E}$ contains the canonical pre- image of all its step spaces.

An interval valued sequence space $\bar{E}$ is said to be convergence free if $\bar{y}=\left(\bar{y}_{k}\right) \in \bar{E}$ whenever $\bar{x}=\left(\bar{x}_{k}\right) \in \bar{E}$ and $\bar{x}_{k}=\overline{0}$ implies $\bar{y}_{k}=\overline{0}$.

Throughout the paper, $p=\left(p_{k}\right)$ is a sequence of bounded strictly positive numbers.

Esi[1] define the following interval valued sequence space:

$$
\bar{\ell}(p)=\left\{\bar{x}=\left(\bar{x}_{k}\right): \sum_{k=1}^{\infty}\left[d\left(\bar{x}_{k}, \overline{0}\right)\right]^{p_{k}}<\infty\right\},
$$

for $p_{k}=1$ for all $k \in N$, we have

$$
\bar{\ell}(p)=\left\{\bar{x}=\left(\bar{x}_{k}\right): \sum_{k=1}^{\infty}\left[d\left(\bar{x}_{k}, \overline{0}\right)\right]<\infty\right\} .
$$

Kizmaz [12] defined the sequence space for crisp set. The concept further generalized by Tripathy and Esi [12] as follows:

Let $m>0$ be an integer then $Z_{1}\left(\Delta_{m}\right)=\left\{\left(\bar{x}_{k}\right) \in w:\left(\Delta_{m} x_{k}\right) \in Z_{1}\right\}$, for $Z_{1}=\ell_{\infty}, c$ and $c_{0}$. Where $\Delta_{m} x_{k}=x_{k}-x_{k+m}$, for all $k \in N$ and they showed that these are Banach spaces under the norm $\|x\|_{\Delta_{m}}=\sum_{r=1}^{m}\left|x_{r}\right|+$ $\sup _{k}\left|\Delta_{m} x_{k}\right|$. For $m=1$, the sequence spaces $\ell_{\infty}(\Delta), c(\Delta)$ and $c_{0}(\Delta)$ are studied by Kizmaz [12]. 
In this paper we introduce the difference operator for sequence of interval numbers generalized by Tripathy and Esi [22] as follows:

Let $\bar{x}=\left(\bar{x}_{k}\right)$ be a sequence of interval numbers and $p=\left(p_{k}\right)$ is a sequence of bounded strictly positive numbers. Let $m \geq 0$ be an integer then

$Z\left(\Delta_{m}\right)=\left\{\left(\bar{x}_{k}\right) \in w^{i}:\left(\Delta_{m} \bar{x}_{k}\right) \in Z\right\}$ for $Z=\bar{\ell}_{p}\left(\Delta_{m}\right), \bar{c}(p)\left(\Delta_{m}\right)$ and $\overline{c_{0}}(p)\left(\Delta_{m}\right)$, where $\Delta_{m} x_{k}=x_{k}-x_{k+m}$, for all $k \in N$.

\section{Main Results}

Theorem 2.1: The sequence spaces $\bar{\ell}(p)\left(\Delta_{m}\right), \bar{c}(p)\left(\Delta_{m}\right)$ and $\overline{c_{0}}(p)\left(\Delta_{m}\right)$ are complete metric space with respect to the metric defined by

$$
\rho(\bar{x}, \bar{y})=\sum_{k=1}^{\infty}\left[d\left(\bar{x}_{k}, \bar{y}_{k}\right)\right]^{p_{k}}+\sup _{k}\left[d\left(\Delta_{m} \bar{x}_{k}, \Delta_{m} \bar{y}_{k}\right)\right]
$$

Proof: Let $\left(\bar{x}^{i}\right)$ be a Cauchy sequence in $\bar{\ell}(p)\left(\Delta_{m}\right)$ such that $\bar{x}^{i}=\left(\bar{x}_{k}^{i}\right)=\left(\bar{x}_{1}^{i}, \bar{x}_{2}^{i}, \bar{x}_{3}^{i}, \ldots\right) \in \bar{\ell}(p)\left(\Delta_{m}\right)$ for each $i \in N$. Then for a given $\varepsilon>0$, there exists $n_{0} \in N$, such that

$$
\rho\left(\bar{x}^{i}, \bar{x}^{j}\right)=\sum_{k=1}^{\infty}\left[d\left(\bar{x}_{k}^{i}, \bar{x}_{j}^{i}\right)\right]^{p_{k}}+\sup _{k} d\left(\Delta_{m} \bar{x}_{k}^{i}, \Delta_{m} \bar{x}_{k}^{j}\right)<\varepsilon, \text { for all } i, j \geq n_{0}
$$

Then

$$
\begin{gathered}
\sum_{k=1}^{\infty}\left[d\left(\bar{x}_{k}^{i}, \bar{x}_{k}^{j}\right)\right]^{p_{k}}<\varepsilon, \text { for all } i, j \geq n_{0} \\
\Rightarrow d\left(\bar{x}_{k}^{i}, \bar{x}_{k}^{j}\right)<\varepsilon, \text { for all } i, j \geq n_{0} \text { and for all } k \in N . \\
\Rightarrow\left(\bar{x}_{k}^{i}\right) \text { is a Cauchy sequence in } R \text { and for all } k \in N .
\end{gathered}
$$

$\Rightarrow\left(\bar{x}_{k}^{i}\right)$ Converges in $R$ and for all $k \in N$ as $R$ is a Banach space. 
Let ${ }_{j} \lim _{k}^{j}=\bar{x}_{k}$ (say) for each $k \in N$ and $\bar{x}=\left(\bar{x}_{k}\right)$.

From definition (2.1) we have

$$
d\left(\Delta_{m} \bar{x}_{k}^{i}, \bar{x}_{k}^{j}\right)<\varepsilon, \text { for } i, j \geq n_{0} \text { and for } k \in N .
$$

$\Rightarrow\left(\Delta_{m} \bar{x}_{k}^{j}\right)$ is a Cauchy sequence in $R$ for all $k \in N$.

$\Rightarrow\left(\Delta_{m} \bar{x}_{k}^{j}\right)$ converges in $R$ for all $k \in N$.

Let $\lim _{j} \Delta_{m} \bar{x}_{k}^{j}=\bar{y}_{k}$, for each $k \in N$.

Since ${ }_{j} \lim _{k} \bar{x}_{k}^{j}=\bar{x}_{k}$, for each $k \in N$, therefore ${ }_{j}^{\lim _{j}} \bar{x}_{k}^{j}=\bar{x}_{k}$ exist for each $k \in N$.

We have

$$
\lim _{j \rightarrow \infty} \sum_{k=1}^{\infty} d\left(\bar{x}_{k}^{i}, \bar{x}_{k}^{j}\right)=\sum_{k=1}^{\infty} d\left(\bar{x}_{k}^{i}, \bar{x}_{k}\right)<\varepsilon, \text { for all } i \geq n_{0}
$$

and

$\lim _{j \rightarrow \infty} d\left(\left(\bar{x}_{k+m}^{i}-\bar{x}_{k+m}^{j}\right),\left(\bar{x}_{k}^{i}-\bar{x}_{m}^{j}\right)\right)=d\left(\left(\bar{x}_{k+m}^{i}-\bar{x}_{k+m}\right),\left(\bar{x}_{k}^{i}-\bar{x}_{m}\right)\right)<\varepsilon$,

for all $i \geq n_{0}$ and $k \in N$.

Hence for all $i \geq n_{0}, \quad \quad \sup _{k} d\left(\Delta_{m} \bar{x}_{k}^{i}, \Delta_{m} \bar{x}_{k}\right)<\varepsilon$.

Thus we have

$$
\begin{gathered}
\sum_{k=1}^{\infty}\left[d\left(\bar{x}_{k}^{i}, \bar{x}_{k}\right)\right]^{p_{k}}+\sup _{k}\left(d\left(\Delta_{m} \bar{x}_{k}^{i}, \Delta_{m} \bar{x}_{k}\right)\right)<2 \varepsilon, \text { for all } i \geq n_{0} . \\
\Rightarrow \rho\left(\bar{x}^{i}, \bar{x}\right)<2 \varepsilon, \text { for all } i \geq n_{0} .
\end{gathered}
$$

i.e. $\bar{x}^{i} \rightarrow \bar{x}$, as $i \rightarrow \infty$ in $\bar{\ell}(p)\left(\Delta_{m}\right)$.

And for $i \geq n_{0}$,

$$
\sup _{k}\left(d\left(\Delta_{m} \bar{x}_{k}, \overline{0}\right)\right) \leq \sup _{k}\left(d\left(\Delta_{m} \bar{x}_{k}, \Delta_{m} \bar{x}_{k}^{i}\right)\right)+\sup _{k}\left(d\left(\Delta_{m} \bar{x}_{k}^{i}, \overline{0}\right)\right)<\infty .
$$

This completes the proof. 
Theorem 2.2: The sequence spaces $\bar{\ell}(p)\left(\Delta_{m}\right), \bar{c}(p)\left(\Delta_{m}\right)$ and $\bar{c}_{0}(p)\left(\Delta_{m}\right)$ are solid.

Proof: Let $\bar{x}=\left(\bar{x}_{k}\right) \in \bar{\ell}(p)\left(\Delta_{m}\right)$ and $\bar{y}=\left(\bar{y}_{k}\right) \in \bar{\ell}(p)\left(\Delta_{m}\right)$ be interval valued sequences such that $\left|\bar{y}_{k}\right| \leq\left|\bar{x}_{k}\right|$ for all $k \in N$.

Then

$$
\sum_{k=1}^{\infty}\left[d\left(\Delta_{m} \bar{x}_{k}, \overline{0}\right)\right]^{p_{k}}<\infty
$$

and

$$
\sum_{k=1}^{\infty}\left[d\left(\Delta_{m} \bar{y}_{k}, \overline{0}\right)\right]^{p_{k}} \leq \sum_{k=1}^{\infty}\left[d\left(\Delta_{m} \bar{x}_{k}, \overline{0}\right)\right]^{p_{k}}<\infty .
$$

Thus $\bar{y}=\left(\bar{y}_{k}\right) \in \bar{\ell}(p)\left(\Delta_{m}\right)$ and hence $\bar{\ell}(p)\left(\Delta_{m}\right)$ is solid.

This complete the proof.

Theorem 2.3: The sequence spaces $\bar{\ell}(p)\left(\Delta_{m}\right), \bar{c}(p)\left(\Delta_{m}\right)$ and $\bar{c}_{0}(p)\left(\Delta_{m}\right)$ are not convergence free.

Proof: Let $m=2$, we consider the interval sequence $\bar{x}=\left(\bar{x}_{k}\right)$ as follows

$$
\bar{x}_{k}=\left[\frac{-1}{k^{2}}, 0\right], \Delta_{2} \bar{x}=\left[\frac{-1}{k^{2}}, \frac{1}{(k+2)^{2}}\right], \text { for all } k \in N .
$$

Then, for $p_{k}=1$

$$
\sum_{k=1}^{\infty}\left[d\left(\Delta_{2} \bar{x}_{k}, \overline{0}\right)\right]<\sum_{k=1}^{\infty}\left(\frac{1}{k^{2}}\right)<\infty .
$$

Thus $\bar{x}=\left(\bar{x}_{k}\right) \in \bar{\ell}(p)\left(\Delta_{m}\right)$.

Now let us define $\bar{y}=\left(\bar{y}_{k}\right)$ as follows

$$
\bar{y}_{k}=\left[-k^{2}, 0\right], \text { then } \Delta_{2} \bar{y}_{k}=\left[-k^{2},(k+2)^{2}\right], \text { for all } k \in N .
$$

Then

$$
\sum_{k=1}^{\infty}\left[d\left(\Delta_{2} \bar{y}_{k}, \overline{0}\right)\right] \leq \sum_{k=1}^{\infty}(k+2)^{2}=\infty .
$$

Thus $\bar{y}=\left(\bar{y}_{k}\right) \notin \bar{\ell}(p)\left(\Delta_{m}\right)$.

Hence $\bar{\ell}(p)\left(\Delta_{m}\right)$ is not convergence free.

This completes the proof. 


\section{References}

[1] A. Esi, Strongly almost $\lambda$-convergence and statistically almost $\lambda$ convergence of interval numbers, Scientia Magna, 7 (2), pp. 117-122, (2011).

[2] A.Esi, Strongly $\lambda$ - summable sequences of interval numbers, Int. Jour. Sc. Env. Tech., 5 (6), pp. 4643-4648, (2016).

[3] A. Esi, $\lambda$ - Sequence spaces of interval numbers, Appl. Math. Inf. Sci., 8 (3), pp. 1099-1102, (2014).

[4] A. Esi, A new class of interval numbers, Jour. Qafqaz Univ., Math. Comp. Sc., 31, pp. 98-102, (2011).

[5] A. Esi, Lacunary sequence spaces of interval numbers, Thai Jour. Math. 10 (2), pp. 445-451, (2012).

[6] A. Esi, Double lacunary sequence spaces of double sequence of interval numbers, Proyecciones Jour. Math., 31 (1), pp. 297-306, (2012).

[7] A. Esi, Statistical and lacunary statistical convergence of interval numbers in topological groups, Acta Sc. Tech., 36 (3), pp. 491-495, (2014).

[8] A. Esi and N. Braha, On asymptotically $\lambda$-statistical equivalent sequences of interval numbers, Acta Sc. Tech., 35 (3), pp. 515-520, (2013).

[9] A. Esi and A. Esi, Asymptotically lacunary statistically equivalent sequences of interval numbers, Int. Jour. Math. Appl., 1 (1), pp. 4348, (2013).

[10] A. Esi and B. Hazarika, Some ideal convergence of double $\wedge$-interval number sequences defined by Orlicz function, Global Jour. Math. Anal., 1 (3), pp. 110-116, (2013).

[11] A. Esi and M. N. Çatalbaş, Some sequence spaces of interval numbers defined by Orlicz functions, Proc. Jangjeon Math. Soc., 20 (1), pp. 35-41, (2017).

[12] H. Kizmaz, On certain sequence spaces. Canad. Math. Bull., 24(2), pp. 168-176, (1981). 
[13] Kuo-Ping Chiao, Fundamental properties of interval vector max-norm, Tamsui Oxford Jour. Math., 18(2), pp. 219-233, (2002).

[14] M. Şengnl and A. Eryilmaz, On the sequence spaces of interval numbers, Thai Jour. Math., 8(3), pp. 503-510, (2010).

[15] P. S. Dwyer, Linear Computation, New York, Wiley, (1951).

[16] P. S. Dwyer, Errors of matrix computation, simultaneous equations and eigenvalues, National Bureu of Standarts, Applied Math. Series, 29, pp. 49-58, (1953).

[17] P. S. Fischer, Automatic propagated and round-off error analysis, paper presented at the 13th National Meeting of the Association of Computing Machinary, June (1958).

[18] R. E. Moore, Automatic Error Analysis in Digital Computation, LSMD-48421, Lockheed Missiles and Space Company, (1959).

[19] R. E. Moore and C. T. Yang, Interval Analysis I, LMSD-285875, Lockheed Missiles and Space Company, (1962).

[20] R. E. Moore and C. T. Yang, Theory of an interval algebra and its application to numeric analysis, RAAG Memories II, Gaukutsu Bunken Fukeyu-kai, Tokyo, (1958).

[21] S. Markov, Quasilinear spaces and their relation to vector spaces, Elect. Jour. Math. Comp., 2(1) (2005).

[22] B. C. Tripathy and A. Esi, A new type of difference sequence spaces. Int. Jour. Sci. Tech., 1 (1), pp. 11-14, (2006).

[23] Zadeh. L.A., Fuzzy sets. Inf. Control, 8(3), pp. 338-353, (1965).

\author{
Achyutananda Baruah \\ Department of Mathematics \\ North Gauhati College \\ Guwahati, Assam \\ India \\ e-mail : achyutanandabaruah@gmail.com
}

and 
Amar Jyoti Dutta

Department of Mathematics

Pragjyotish College

Guwahati, Assam

India

e-mail : amar_iasst@yahoo.co.in 